\title{
Reliability Demonstration Approach for Advanced Stirling Radioisotope Generator
}

\author{
Chuong $\mathrm{Ha}^{1}$ \\ Lockheed Martin Space Systems, Sunnyvale, CA, 94089 \\ Edward Zampino ${ }^{2}$ \\ NASA Glenn Research Center, Cleveland, OH, 44135 \\ Barry Penswick ${ }^{3}$ \\ Sunpower, Athens, $\mathrm{OH}, 45701$ \\ and \\ Michael Spronz ${ }^{4}$ \\ Sest Inc., Cleveland, OH, 44135
}

\begin{abstract}
Developed for future space missions as a high-efficiency power system, the Advanced Stirling Radioisotope Generator (ASRG) has a design life requirement of $14 \mathrm{yr}$ in space following a potential storage of $3 \mathrm{yr}$ after fueling. In general, the demonstration of long-life dynamic systems remains difficult in part due to the perception that the wearout of moving parts cannot be minimized, and associated failures are unpredictable. This paper shows a combination of systematic analytical methods, extensive experience gained from technology development, and well-planned tests can be used to ensure a high level reliability of ASRG. With this approach, all potential risks from each life phase of the system are evaluated and the mitigation adequately addressed. This paper also provides a summary of important test results obtained to date for ASRG and the planned effort for system-level extended operation.
\end{abstract}

\section{Nomenclature}

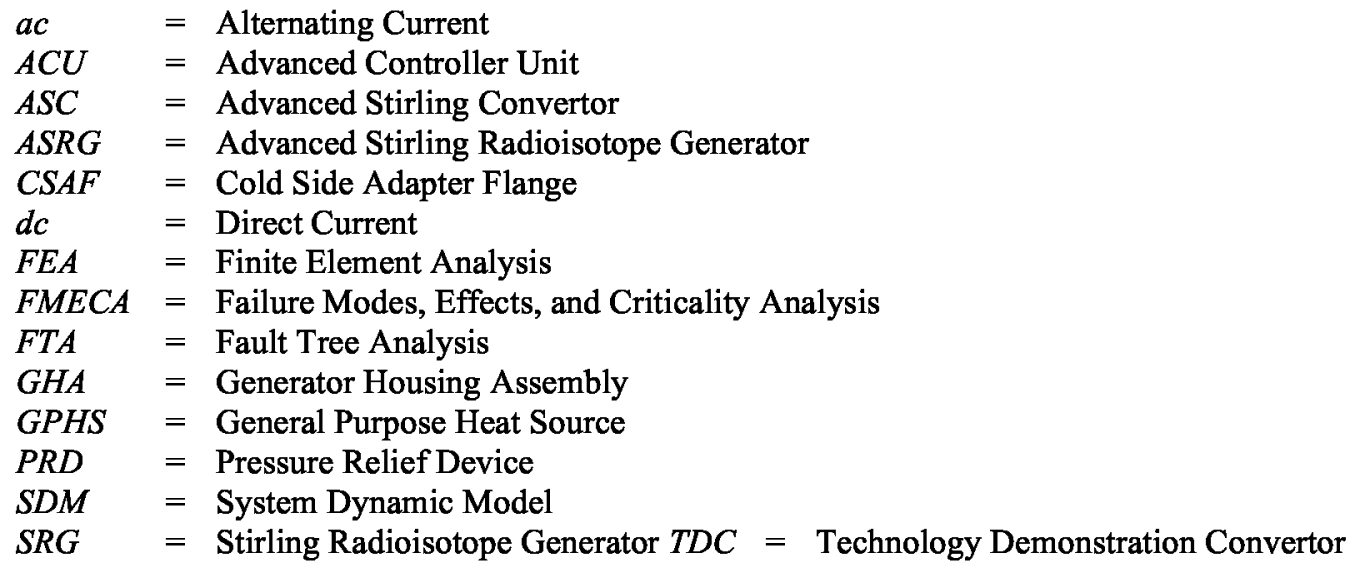

${ }^{1}$ Reliability Engineer Sr. Staff, 1111 Lockheed Martin Dr., MS O7/LMS B/157, Sunnyvale, CA 94089.

${ }^{2}$ Reliability Engineering Lead, Program and Project Assurance, 21000 Brookpark Rd., MS 5-4, Cleveland, OH 44135.

${ }^{3}$ Reliability Consultant, Sunpower Inc., 182 Mill St., Athens, OH 45701.

${ }^{4}$ QA Engineer Sr., Sest Inc., 18151 Jefferson Park Road, Suite 101, Middleburg Heights, OH 44130.

American Institute of Aeronautics and Astronautics 


\section{Introduction}

$\mathrm{T}$

HE ASRG is being developed by the Department of Energy and NASA ${ }^{1}$ as a highly efficient power system for potential use in future space exploration missions, including deep space missions and surface applications. With the engineering development phase complete in $2008,{ }^{2}$ the qualification design effort is well underway for flight readiness. One of the key requirements of the ASRG is the capability to perform its functions for a 17-yr design life with a probability of success of at least $90 \%$. The required design life consists of a $14 \mathrm{yr}$ operation in space following a potential storage of $3 \mathrm{yr}$ in controlled environments after fueling. While this design life is typical for deep space mission systems as well as for commercial satellites, reliability demonstration for onboard continuously running mechanisms remains a key challenge. For example, attitude control subsystems of spacecraft such as gyros and momentum wheels could reach between 3000 to $8000 \mathrm{rpm}$. In the case of the Advanced Stirling Convertor (ASC) units of the ASRG, the moving free-piston and displacer subsystem operates at about $102 \mathrm{~Hz}$. Thus, for a 17yr design life the total number of cycles is predicted to reach 55 billion $\left(5.5 \times 10^{10}\right)$. Clearly, a system testing at such long duration and high cycle levels is impractical. Instead, extensive knowledge accumulated throughout the development years has led to these key observations:

- ASRG reliability demonstration cannot be based exclusively on a single, classic life test but must rely on a combination of analytical models and alternative tests.

- Moving parts only represent one aspect of the complete ASRG functionality. Any potential life-limiting risks from moving parts as well as other subsystems must be thoroughly understood and adequately mitigated.

- Random and wearout failures are applicable to specific life phases of the design and must be tackled differently.

- Capability to simulate subsystem interface and performance in all expected operating conditions is essential to ensure mission success.

- Well-planned qualification and accelerated testing at component or subsystem levels can also provide valuable data for reliability modeling and demonstration.

\section{ASRG Design}

As shown in Fig. 1, the overall ASRG system consists of the Generator Housing Assembly (GHA) and the Advanced Controller Unit (ACU). The GHA encloses two ASCs that are mounted in opposite directions, the associated General Purpose Heat Source (GPHS) units, the heat support assemblies, and the thermal insulation material segments. For ground operation, the GHA is filled with inert gas to protect the GPHS graphite material from oxidation. During launch ascent, the barometric pressure decrease will trigger a diaphragm puncture inside the Pressure Relief Device (PRD), evacuating the inert gas.

As a free-piston Stirling convertor, the ASC is designed to convert the GPHS heat into alternating current (ac) electrical power. Helium is used as the working gas, hermetically contained within the ASC pressure vessel. At a frequency of about $102 \mathrm{~Hz}$, the displacer and piston assemblies reciprocate with helium pressure variations between the expansion and compression spaces. The ac electrical power is produced from the linear alternator that consists of laminations, alternator coil, and moving magnets. Inside the ASC, a high-porosity matrix made of corrosionresistant material allows heat recovery of the working gas between expansion and compression cycles. The Cold Side Adapter Flange (CSAF) provides both structural connection of the ASC to the GHA and a heat rejection path to the outer shell and radiation fins. The electrical power generated by the ASC is sent to the controller via two hermetic feedthroughs.

With the possibility to be mounted remotely inside the spacecraft or on the rover, the ACU provides the operational control of the ASCs and the system telemetry signals. The ACU is an active-power-factor design with a fixed-frequency operating point. The ACU synchronizes the ASCs to minimize the dynamic vibration and convert the ac to direct current (dc) power. In case of dc bus off-nominal conditions, the ACU regulates the excess power with the Shunt Dissipation Unit, mounted at the GHA end to take advantage of a better heat rejection capability. 


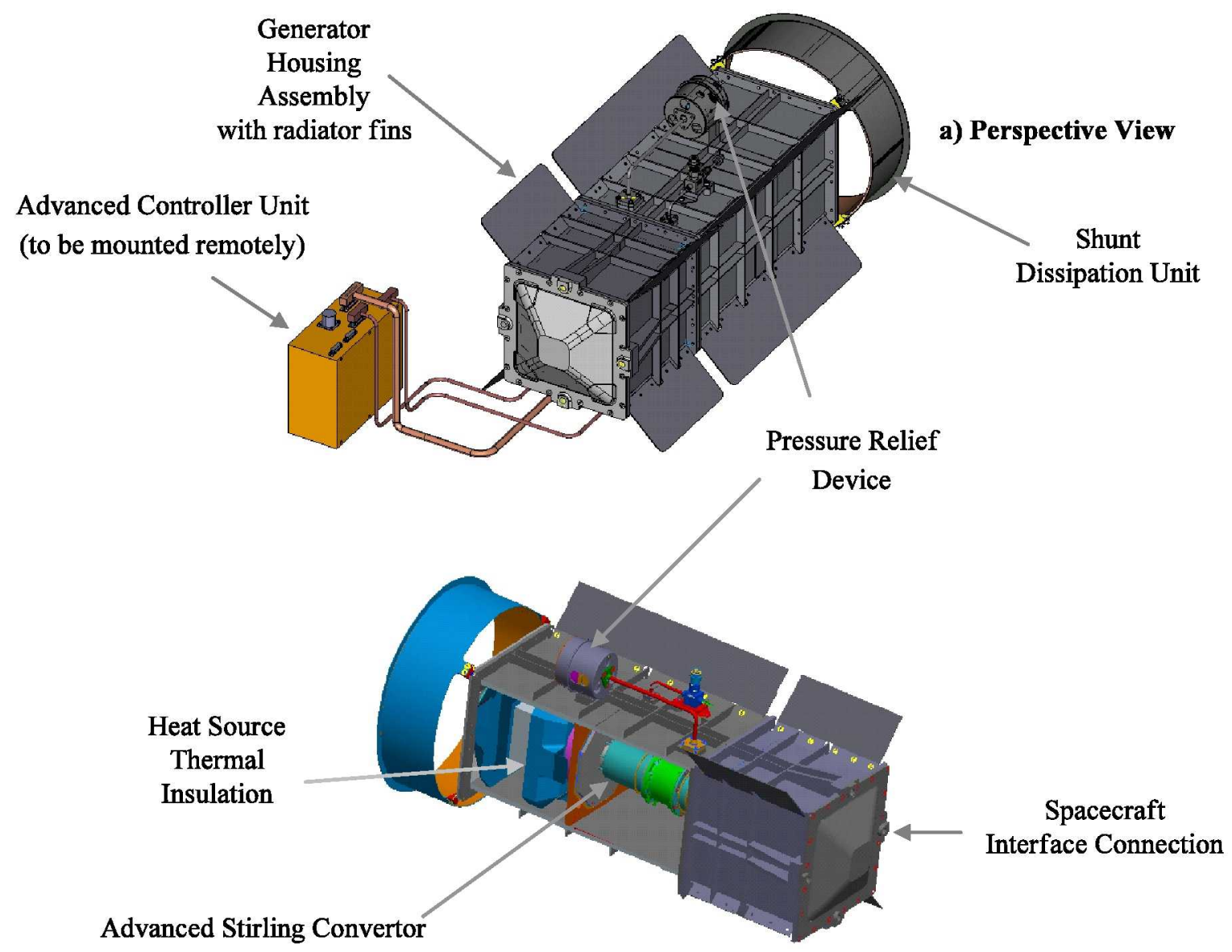

b) Cut-away view of Generator Housing Assembly

Figure 1. ASRG Subsystems and Components

\section{Tasks of the ASRG Reliability Demonstration Approach}

The ASRG reliability demonstration activities can be grouped into four major categories as depicted in Fig. 2. Those categories are

a) Risk identification using expert solicitation and Failure Modes, Effects, and Criticality Analysis (FMECA) to provide an early identification of the potential failure modes

b) Risk quantification using reliability modeling to quantify the risks at component and system levels

c) Risk mitigation with applicable tests and analyses

d) Final risk integration to provide an assessment of the system-level reliability

In this context, risk is defined as being applicable to reliability since it relates directly to the severity and likelihood of failure modes. In general, the four task categories are performed in sequence but can be iterated as necessary. Details of each task category are provided in the following sections. 


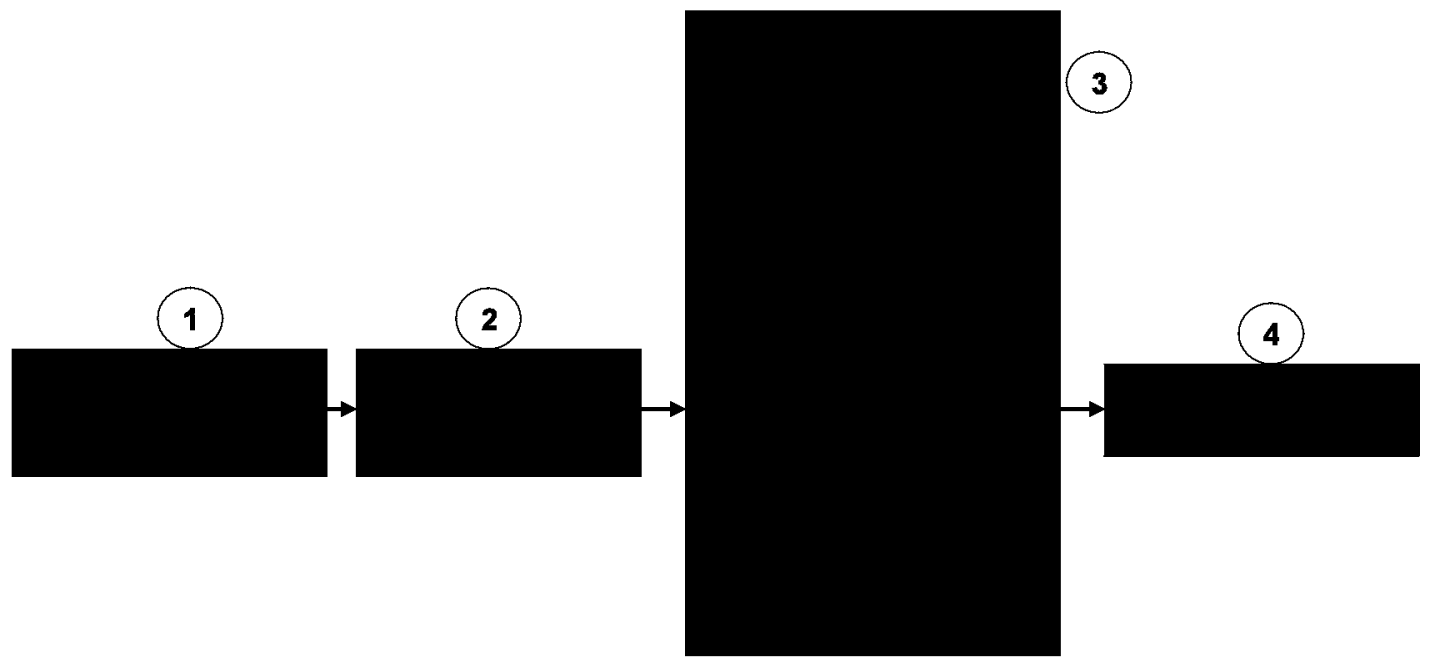

Figure 2. Tasks of the ASRG Reliability Demonstration

\section{A. Risk Identification}

At the beginning of the ASRG program, when design details were still evolving in many areas, the development team decided that a risk identification process must be implemented and maintained regularly. In steps with the design activity progress, this effort would identify any life-limiting risks and keep track of mitigation actions. The risk identification process has benefited significantly from inputs of experts at NASA Glenn Research Center, NASA Jet Propulsion Laboratory, Sunpower, and Lockheed Martin. Insights from the designers also formed a valuable understanding of the system characteristics. Using the FMECA technique, the potential failure modes of each component and associated impacts were systematically identified. The failure mode severity is ranked based on three simple qualitative levels: 1) no impacts on power performance; 2) reduced or degraded power performance; and 3) imminent or catastrophic loss of power performance. In terms of likelihood ranking, the five-level system from MIL-STD-1629A $\mathrm{A}^{3}$ was adopted to allow the necessary resolution. In this system, the highest value (5) represents a frequent occurrence while the lowest value (1) represents an unlikely one. The failure mode criticality is then calculated as Severity times Likelihood. As output, the FMECA provides a criticality matrix that involves all components in the system. Critical items considered as potential single point failures are clearly listed for monitoring and mitigating.

\section{B. Risk Quantification With Reliability Models}

Following the risk identification process with FMECA, we generated the reliability models for both components and the full system to quantify the risks. Usually, the component reliability models are not needed because the derivation of failure probability relies on analysis of existing test results and usage history. Most of the electronic parts have established standard methods and vendor database for their qualification analysis. However, when the design is new or only limited test data are available, some iteration is required. Several reliability component models must have initially utilized similar equipment data and then gradually switched to actual data as qualification or accelerated tests were completed. In other instances, the component reliability models were generated using Finite Element Analysis (FEA) for stress-strength interference prediction. Most of these FEA models applied to structural components. When the design data was available, the component reliability models took into account the relevant uncertainties from design and operating condition parameters. As data from the qualification and accelerated tests became available, the stress-strength interference predictions were updated accordingly.

At the system level, we preferred the Fault Tree Analysis (FTA) model to integrate the results obtained from the component analysis process described previously. In order to generate the fault tree logic, a review of each subsystem FMECA (ACU, ASC, and GHA) and the system spec requirements was necessary. Information from the FMECA provides input to build the basic failure events and the spec requirements to construct the fault logic. The FTA model details were updated as needed providing the risk drivers and contributions from components and subsystems at periodic reviews. 


\section{Component and Subsystem Accelerated Tests}

Many of the ASRG components are qualified with accelerated testing. These tests not only validate the design margins but also provide inputs to the reliability analyses. However, due to the fundamental operating characteristics of the free piston-linear alternator configuration of the ASC - it is extremely difficult to carry out the classical approach of employing various accelerated testing at the full system. The primary cause of this limitation is the relatively narrow operating envelope of the Stirling engine from the viewpoint of operating speed (frequency), mechanical loads (pressure), electrical loads (alternator capabilities), and temperatures (heater head creep, magnet degradation, etc.), leaving little if any parameters available for system accelerated testing.

To overcome this fact, the development team has employed risk mitigation effort for a wide range of component tests to define their key durability and reliability characteristics under normal and extreme load conditions. The following table provides a set of such testing efforts.

Table 1. Component Accelerated Tests.

\begin{tabular}{|c|c|c|}
\hline Component Type & Purpose & Results \\
\hline Heater head & $\begin{array}{l}\text { Verify creep rate at } 1.4 \text { times } \\
\text { nominal peak internal pressure } \\
\text { and } 869^{\circ} \mathrm{C} \text { max. temperature }\end{array}$ & $\begin{array}{l}\text { Interim measured creep rate at half of } \\
\text { predicted value }\end{array}$ \\
\hline Heater head & $\begin{array}{l}\text { Permeability test of thin wall } \\
\text { specimens at } 850^{\circ} \mathrm{C} \text { and } 6 \% \\
\text { strain }\end{array}$ & No leakage found \\
\hline Fasteners & $\begin{array}{l}\text { Use destruction torque to verify } \\
\text { nut factor and joint load capacity }\end{array}$ & $\begin{array}{l}\text { Each fastener type was tested with } 30 \\
\text { samples-high strength results obtained }\end{array}$ \\
\hline Organics & $\begin{array}{l}\text { Magnet bonding material aging } \\
\text { characterization with exposure } \\
\text { up to } 1.8 \text { times nominal } \\
\text { operating temperature }\end{array}$ & $\begin{array}{l}\text { No significant changes at } 2 \mathrm{yr} \text { aging in } \\
\text { terms of weight change and mechanical } \\
\text { properties }\end{array}$ \\
\hline Organics & $\begin{array}{l}\text { Gamma irradiation tests of } \\
\text { organics used in the ASC at } 5 \text {, } \\
10 \text {, and } 15 \mathrm{Mrad}\end{array}$ & $\begin{array}{l}\text { Large margins for operating conditions } \\
\text { confirmed-ongoing tests }\end{array}$ \\
\hline Linear alternator & $\begin{array}{l}\text { Determine maximum } \\
\text { temperature for onset } \\
\text { demagnetization }\end{array}$ & $\begin{array}{l}\text { The onset temperature of nonrecoverable } \\
\text { degradation determined }\end{array}$ \\
\hline Displacer spring & Verify fatigue endurance & $\begin{array}{l}\text { High margin results obtained with step- } \\
\text { stress tests - additional tests with constant } \\
\text { stress method ongoing spring material } \\
\text { to be tested at giga cycles level }\end{array}$ \\
\hline Power feedthrough & $\begin{array}{l}\text { Verify hermeticity with } \\
\text { increasing temperature up to } 4 \\
\text { times nominal operating } \\
\text { temperature }\end{array}$ & $\begin{array}{l}\text { Five different model samples tested; } \\
\text { ceramic seal selected over glass; } \\
\text { additional tests with elevated temperature } \\
\text { and axial loads also show very high } \\
\text { margins }\end{array}$ \\
\hline Insulation material & $\begin{array}{l}\text { Verify long-term shrinkage of } \\
\text { samples at max. temperature } \\
950^{\circ} \mathrm{C}\end{array}$ & $\begin{array}{l}\text { Interim data at } 8000 \mathrm{hr} \text { collected-- } \\
\text { shrinkage ranging from } 2 \text { to } 10 \% \\
\text { depending on insulation layer temperature }\end{array}$ \\
\hline
\end{tabular}

In Table 1, it is significant to note that

- Most of the ASC fasteners are $2 \mathrm{~mm}$ in size; therefore, extremely small by conventional structural standards and in a number of cases represent potential single point failures. This size characteristic required a master buy of fasteners along with extensive testing for both the fastener material as well as the installation torque for all operating conditions. This latter effort required a careful test planning to simulate mechanical joints of the convertor since a wide range of materials are employed.

- Linear alternator design limits are critical to defining the safe operating conditions for this critical component. A unique test rig, called a Hot Alternator Test Rig (Fig. 4), was employed in this process. The test rig allows the test ASC alternator to be driven at various amplitudes and power levels while 
being heated to selected temperatures. These temperatures allowed definition of the onset of magnet degradation in the actual ASC alternator configuration and structural limits for the various materials employed in the hardware.

- Displacer spring is one critical component of the ASC moving parts, operating at a nominal frequency of $102 \mathrm{~Hz}$. The spring design has benefited significantly from the extensive experience at Sunpower with the cryocooler models, one of which was flight proven. ${ }^{4}$ Testing of multiple springs over a wide range of stress conditions with the step-stress method has provided a good margin for the predicted spring reliability. To reduce the uncertainty of the fatigue threshold, an additional set of tests with the constant-stress method is currently being performed. Due to the extremely large number of stress cycles $\left(5.5 \times 10^{10}\right)$ experienced by this component, a test plan is also underway to use a high- frequency shaker to reach or even exceed the full design cycles on spring material samples.

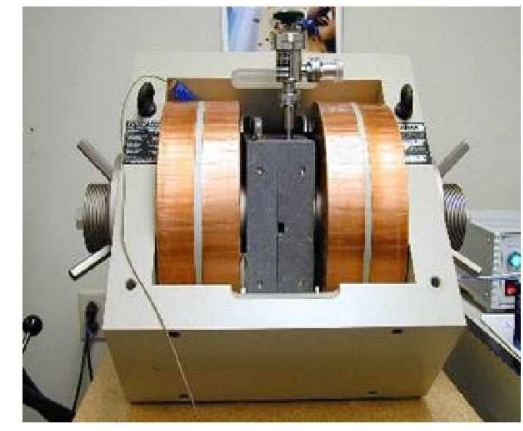

Figure 3. Magnet Aging Test m.-1.....

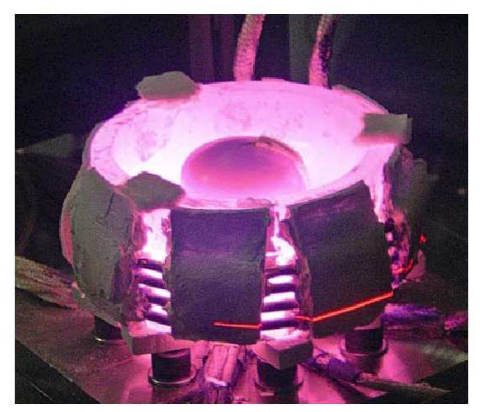

Figure 5. Heater Head Benchmark ruman Dota Tout

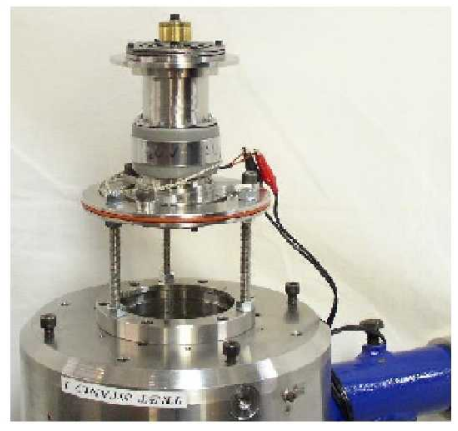

Figure 4. Hot Alternator Test

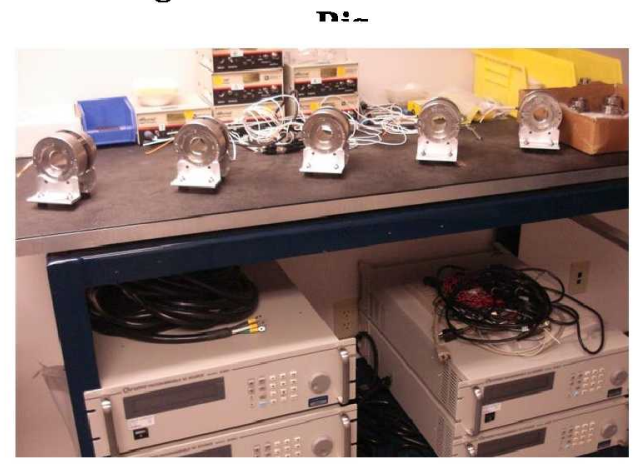

Figure 6. Displacer Spring Test Station With Linear Motor Drives and Controllers

\section{Integrated Simulation Model}

One aspect of the ASRG reliability sensitivity is the interface between the ASC and ACU. The ACU main requirements are to convert the output power from ac to $\mathrm{dc}$ and to synchronize the convertors in opposite direction to minimize mechanical vibration. As an autonomous system, the ACU is also expected to generate adjustment commands to adapt to changes such as usage load and operating temperature variations. Designed as single fault tolerant, the ACU relies on an $\mathrm{N}+1$ redundant scheme, using a standby control card as instant backup to any of the two primary control cards. While the ACU design requirements must be complete for all operating conditions and its functionality thoroughly tested, the addition of an analytical integrated model has offered many advantages in simulating nominal and off-nominal condition of interest. Known as System Dynamic Model (SDM) ${ }^{5}$, a computerized model of the ASC-ACU interface was developed at NASA Glenn to simulate conditions such as performance stability, system frequency response, and fault simulation with controller card switchover. Specifically for the latter case, SDM provided the maximum allowed time for switching between the primary and backup controller cards as a function of a buffer load resistance. With the capability to simulate both ASC and ACU 
response parameters, SDM will allow validation of the ACU control approach for conditions that the mission Concept of Operations could envision.

\section{E. Risk Mitigation With Subsystem Durability Tests}

The objective of the durability tests is to demonstrate the ASC robustness in overstress conditions. As a preferred approach, we plan to overstress the convertor but avoid damaging so that an extended operation test can be performed afterwards. This implies a careful planning in stress amplitudes and application timing during the test. Overall, the sequential tests for the ASC when subjected to the durability test include

- $\quad$ Acceptance tests at delivery

- Run-in test between 500 to $1000 \mathrm{hr}$ to characterize basic performance

- Sequence of overstress tests and associated post-test performance checks

- Extended operation to demonstrate long life

We considered 10 types of tests that could subject the ASCs to overstress conditions. Table 2 provides the list of tests and associated priority and rationale. After reviewing other concurrent tests and existing results, the priority was assigned to each of the considered tests. Plans are underway to execute all the tests with high priority.

Table 2. ASRG Durability Tests

\begin{tabular}{|l|l|l|}
\hline \multicolumn{1}{|c|}{ Overstress Parameter } & \multicolumn{1}{|c|}{ Priority } & \multicolumn{1}{c|}{ Rationale } \\
\hline $\begin{array}{l}\text { ASC piston over-stroke } \\
\text { during random vibration }\end{array}$ & High & $\begin{array}{l}\text { - To simulate potential piston collisions during high vibration } \\
\text { conditions; number of over-strokes during test controlled with } \\
\text { commanded amplitude }\end{array}$ \\
\hline $\begin{array}{l}\text { ASC piston over-stroke } \\
\text { during out-of-control time }\end{array}$ & High & $\begin{array}{l}\text { - To simulate prolonged off-nominal conditions such as out of } \\
\text { control or switchover }\end{array}$ \\
\hline Static acceleration & High & $\begin{array}{l}\text { - To simulate high static acceleration during liftoff or landing } \\
\text { - To be tested with centrifugal accelerator }\end{array}$ \\
\hline High temperature cycling & Medium to high & $\begin{array}{l}\text { - To simulate extreme temperature effects on ASC operation } \\
\text { - Potential high stresses for alternator components } \\
\text { - Test could be combined with extended operation }\end{array}$ \\
\hline Piston amplitude & High & $\begin{array}{l}\text { - High stress effects on most ASC components } \\
\text { - Moving parts subjected to accelerated stress factor } \\
\text { - Test could be combined with extended operation }\end{array}$ \\
\hline Startup and shutdown cycles & High & $\begin{array}{l}\text { - To ensure the ASC moving parts robustness during various } \\
\text { ground operation and tests before final fuel loading }\end{array}$ \\
\hline Shock test & Low to medium & $\begin{array}{l}\text { - Shock tests already being planned for system qualification } \\
\text { - Engineering system successfully passed 3000 g shock test }\end{array}$ \\
\hline Random vibration & Low & $\begin{array}{l}\text { - Tests already being planned for system qualification } \\
\text { - Some past tests went to qualification level and beyond }\end{array}$ \\
\hline Radiation & Low & $\begin{array}{l}\text { - Test with whole alternator completed successfully } \\
\text { - Coupons of material are being tested at Mrad level } \\
\text { - Use radiation hardening rating for established electronic parts }\end{array}$ \\
\hline Pressure & Low & $\begin{array}{l}\text { - High values of pressure already being utilized in creep and } \\
\text { permeability tests } \\
\text { - Limited variations allowed for a stable ASC operation }\end{array}$ \\
\hline
\end{tabular}

\section{F. Reliability Experience With Similar Designs}

Like any other technology development project, the ASRG design must go through successive changes and refinements for improvements. ${ }^{6}$ In 2000, the development program initially started with the Technology Demonstration Convertor (TDC) to produce a 110-W Stirling Radioisotope Generator (SRG-110). Then in 2006, under the redirection of the Department of Energy (DOE) and NASA, the program integrated the ASC into the system for higher efficiency, maximizing the system specific power. Extensive knowledge accumulated from the development, testing, or fabrication processes provided the program with valuable information to ensure the system could meet its long life operation goal. 
Particularly for the ASC, with the exception of the high operating temperature components such as the heater head and heat collector, the design represents a relatively straightforward application of the free-piston Stirling technology that has evolved at Sunpower. Various Sunpower commercial cryocooler products incorporate very similar sized planar springs for the displacer, gas bearing for the moving piston, and linear drive motor configuration.

From the lessons learned of the development activities, the key efforts that have provided a strong basis for the system reliability and performance include the following:

- Ensure the regenerator will not generate debris during its long life operation through proven material selection and manufacturing process optimization

- Increase the design margins for high temperature components with selection of high creep endurance material and extensive validation tests

- Stringent acceptance tests of the gas bearing system to ensure noncontacting operating conditions between the moving parts under all conditions experienced by the ASC

- Provide a robust and accurate positioning of the ASC piston during all operating conditions

- Incorporate component material that will not contribute to possible degradation mechanisms

- Improve the robustness of fastener internal joints with high strength material and compatible thermal expansion coefficient

- Analyze and extensively test the unique environment of the launch phase to understand the interface ASC-ACU during high vibrations

- Implement an integrated quality assuranceprogram that covers all aspects of hardware development, manufacture, and testing - this effort is critical since the free-piston linear alternator Stirling convertor could be sensitive to small variations in various component parameters

The following table summarizes the results of these efforts with the characteristics of the program convertors and their accumulated test hours from extended operation. With more ASC convertors entering the test program in support of the flight unit development, it is expected that further insights as well as total accumulated convertors operation hours will increase.

Table 3. Extended Operation Tests at NASA Glenn (as of April 2010)

\begin{tabular}{|l|c|l|l|}
\hline \multicolumn{1}{|c|}{$\begin{array}{c}\text { Convertor } \\
\text { System }\end{array}$} & $\begin{array}{c}\text { Accumulated } \\
\text { Hours Per } \\
\text { Unit }\end{array}$ & Status & \multicolumn{1}{|c|}{ Comments } \\
\hline TDC \#5 and TDC \#6 & $10,000 \mathrm{hr}$ & Stopped & $\begin{array}{l}\text { SRG-110 baseline design-thermal vacuum tested-no } \\
\text { failures }\end{array}$ \\
\hline TDC \#13 and TDC \#14 & $52,725 \mathrm{hr}$ & Ongoing & $\begin{array}{l}\text { SRG-110 baseline design-hermetically sealed after } \\
19,000 \mathrm{hr}-\text { no failures }\end{array}$ \\
\hline TDC \#15 and TDC \#16 & $39,331 \mathrm{hr}$ & Ongoing & $\begin{array}{l}\text { SRG-110 baseline design- -hermetically sealed after 4,000 } \\
\text { hr-no failures }\end{array}$ \\
\hline Total TDC hours & $208,315 \mathrm{hr}$ & \multicolumn{2}{|l|}{} \\
\hline ASC-1HS \#1 & $3,842 \mathrm{hr}$ & Ongoing & Development design \\
\hline ASC-1HS \#4 & $7,824 \mathrm{hr}$ & Ongoing & Development design \\
\hline ASC-0 \#1 and \#2 & $15,378 \mathrm{hr}$ & Stopped & $\begin{array}{l}\text { Development design-piston of ASC-0 \#1 drifted due to } \\
\text { known causes from burn-in tests }\end{array}$ \\
\hline ASC-0 \#3 and \#4 & $17,718 \mathrm{hr}$ & Ongoing & $\begin{array}{l}\text { Development design-piston design improved-no } \\
\text { failures }\end{array}$ \\
\hline ASC-1 \#3 and \#4 & $1,817 \mathrm{hr}$ & Ongoing & Development design \\
\hline Total development ASC & $80,478 \mathrm{hr}$ & \multicolumn{2}{|l|}{} \\
\hline ASC-E \#2 and \#3 & $9,613 \mathrm{hr}$ & Ongoing & ASRG engineering unit system level test with controller \\
\hline ASC-E \#1 & $2,452 \mathrm{hr}$ & Ongoing & Engineering ASC design \\
\hline ASC-E \#4 & $2.398 \mathrm{hr}$ & Ongoing & Engineering ASC design \\
\hline Total ASC hours & $24,076 \mathrm{hr}$ & \multicolumn{3}{|l}{} \\
\hline
\end{tabular}




\section{G. Integrated System Extended Operation}

Traditionally, we use the extended operation (life test) to demonstrate the integrated design has eliminated potential random failures and wearout risks. These failure modes are applicable to the mid- and late-life phases of the system, rather than the early-life phase because infant mortality failures can be mitigated by extensive burn-in and qualification tests. Unlike the durability tests previously described, the extended operation tests drive the integrated system at nominal operating conditions for a target duration or as long as possible. It should be emphasized that life test, while very important, is not the only reliability measure of the system. Other component test data and analytical model results must be considered to arrive at a complete reliability prediction. As to complement other efforts, the focus of the extended operation is also on detecting failure modes and operation anomalies at the system level unforeseen by other analyses and tests.

For ASRG, the 17-yr design life and continuous high cycle level operation have made impractical a full life test on the same test unit. Sensitivity calculations using the Weibull model ${ }^{7}$ to reach the reliability target solely through a zero-failure life test plan, have led to sample size and test time that far exceed any program resources. Moreover, even a relaxation in confidence level (lower than $\mathbf{9 0 \%}$ ) gave little relief on test duration. Instead, we adopt the following approach for life test:

- Since the design has no contacts on moving parts, the random failure modes are assessed as more dominant contributors than the wearout failure modes during the entire system life. Random failures are independent of time and therefore the test duration target can be cumulative from a number of systems under test.

- Based on the recommendation of aerospace standards for moving mechanism, ${ }^{8}$ the cumulative test duration goal is to achieve 1.5 times design life (or $25.5 \mathrm{yr}$ ). For a potential 2015 launch target, this goal is achievable with high confidence, using at least six systems (each with two ASCs), three of which will start in early 2011 , and the remaining in early 2012 . The estimated total accumulated hours from the ASCs of these six systems will reach 38 yr or larger than 2 times design life.

- Considering the insights gained from the existing life tests, the test duration from 4 to $5 \mathrm{yr}$ would be sufficient to uncover any life-limiting risks.

- To address the wearout and degradation concerns, we rely on the accelerated tests of components and durability tests (described previously). For moving parts particularly, even if the traditional life test approach could be carried out, a very large amount of accrued test time might be required due to the nocontact design characteristics. Instead, it is better to perform durability tests that simulate overstress conditions that might lead to the potential rubbing and subsequent debris generation. Certainly, workmanship and quality assurance control of the high dimension tolerances also help to ensure a long life operation.

- For each system in the extended operation life test, we have recommended to use an integrated ASCACU configuration. Even interim versions of the ACU before flight could provide a better understanding of the interface between the two subsystems. The objective of the ACU presence is not about electronic parts life testing but to understand the interface and to characterize all operating conditions.

\section{H. Reliability Prediction With FTA System Model}

The final step of reliability demonstration is to integrate the risks and interpret the results. As a probabilistic analysis, one could use either the Reliability Block Diagram or the FTA technique. In order to have a complete integration of risks derived from test results and analyses described above, we generated a system FTA model to predict the reliability. With sufficient knowledge and relevant test data, the typical FTA is a straightforward and systematic top-down method to provide both the overall system reliability and individual contributions at subsystem or component levels. The FTA model also provides the overall risk ranking and the list of cutsets or potential combination of failure events that lead to the top event. For specific mission phases and scenarios of interest, a series of conditional fault and event trees are necessary to determine the specific risks.

\section{Conclusion}

The reliability demonstration approach for ASRG includes a series of steps that involve both analytical modeling and tests. The extensive knowledge accumulated throughout the development years allows not only a full system characterization but also a practical approach to test the long life requirement. As key to mission success, the risks associated with each life phase of the system must be understood and associated tests adequately planned. We also believe that like any other design, inherit and usage reliability are covered by sound design and good workmanship. 


\section{Acknowledgments}

The work described in this paper was sponsored by the U.S. Department of Energy and the National Aeronautics and Space Administration. The authors wish to acknowledge the contribution and technical collaboration made by other members of the ASRG Reliability Integrated Product Team.

\section{References}

${ }^{1}$ Chan, J., Wood, J. G., and Schreiber, J. G., "Development of Advanced Stirling Radioisotope Generator for Space Exploration,"NASA/TM-2007-214806, 2007.

${ }^{2}$ Chan, J., Hill, D., Hoye, T., and Leland, D., "Development for Advanced Stirling Radioisotope Generator for Planetry Surface and Deep Space Missions," Proceedings of the 6th International Energy Conversion Engineering Conference, AIAA2008-5790, Cleveland, OH, 2008.

${ }^{3}$ Military Standard, "Procedures for Performing a Failure Mode, Effects, and Criticality Analysis," MIL-STD-1629, Rev. A, 1980.

${ }^{4}$ Wood, J. G., Wilson, K., Carroll, C., Matejczyk, D., Soender, E., and Penswick, L. B., "Advanced Stirling Convertor Phase II Achievements and Planned Phase III Efforts," $4^{\text {th }}$ International Energy Conversion Engineering Conference, AIAA, 20064108.

${ }^{5}$ Regan, T., "Free-Piston Stirling Convertor Controller Development at NASA Glenn Research Center," NASA/CR-2004$213038,2004$.

${ }^{6}$ Wong, W. A., Wood, J. G., and Wilson, K., "Advanced Stirling Convertor (ASC)—From Technology Development to Future Flight Product," NASA/TM-2008-215282, 2008.

${ }^{7}$ Abernathy, R. B., "The New Weibull Handboook," Fifth Edition, Abernathy, 2004.

${ }^{8}$ AIAA Standard, "Moving Mechanical Assemblies for Space and Launch Vehicles," AIAA S-114-2005. 